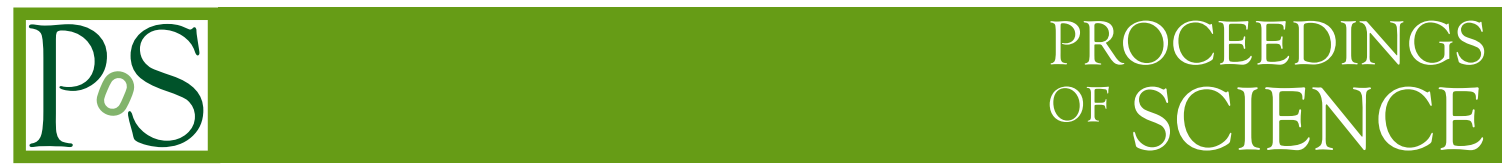

\title{
Internal Target Experiments at the MESA accelerator
}

\author{
Harald Merkel ${ }^{*}$ \\ Johannes Gutenberg University, Mainz \\ E-mail: merkel@kph.uni-mainz.de
}

The Mainz Superconducting Energy Recovery Linac (MESA) is a new accelerator under construction at the University of Mainz. MESA is designed as a recirculating superconducting linear accelerator which provides an external beam with high current and high degree of polarization. In energy recovery mode, MESA can provide even higher internal beam, which is ideal for precision experiments. In this presentation, the physics potential of internal target experiments with such an accelerator is explored.

54th International Winter Meeting on Nuclear Physics

25-29 January 2016

Bormio, Italy

${ }^{*}$ Speaker. 


\section{Introduction}

Electron scattering experiments in the low energy range below the pion threshold are performed since several decades now, increasing considerably our knowledge on nucleon and nuclear structure. The electron is an extremely clean probe, interacting only via the well known electroweak interaction and making electron scattering processes one of the best tools for precision experiments in this energy region.

New developments in accelerator physics now opens the window to a new generation of these precision experiments. Internal beam intensities of several milli-Ampère are available now, making new experiments with gas targets possible where recoil particles with very low energy can be detected with high precision. High degrees of polarization at high beam currents are ideal suited for parity violating experiments. At the same time, the beam quality of the new accelerators permits the use of sensitive large area detectors in close vicinity of the interaction point even at high luminosities.

In Mainz a new accelerator, the Mainz Superconducting Energy Recovery Linac (MESA), is under construction, dedicated to these high precision experiments. The equipment of this accelerator with two high resolution spectrometers (MAGIX) is a unique setup for new electron scattering experiments for nuclear and hadron physics.

In this paper, a few selected examples for experiments which utilize the unique features of this apparatus are presented.

\section{The MESA Accelerator}

Fig. 1 shows the floor plan of the MESA accelerator. MESA is a low energy recirculating linac designed to provide a high intensity beam with high polarization and excellent beam quality for precision experiments.

MESA will be equipped with super-conducting accelerating structures, which will be optimized for high beam current. After an injector section, three recirculating arcs will provide an extracted beam of up to $1 \mathrm{~mA}$ beam current for external experiments.

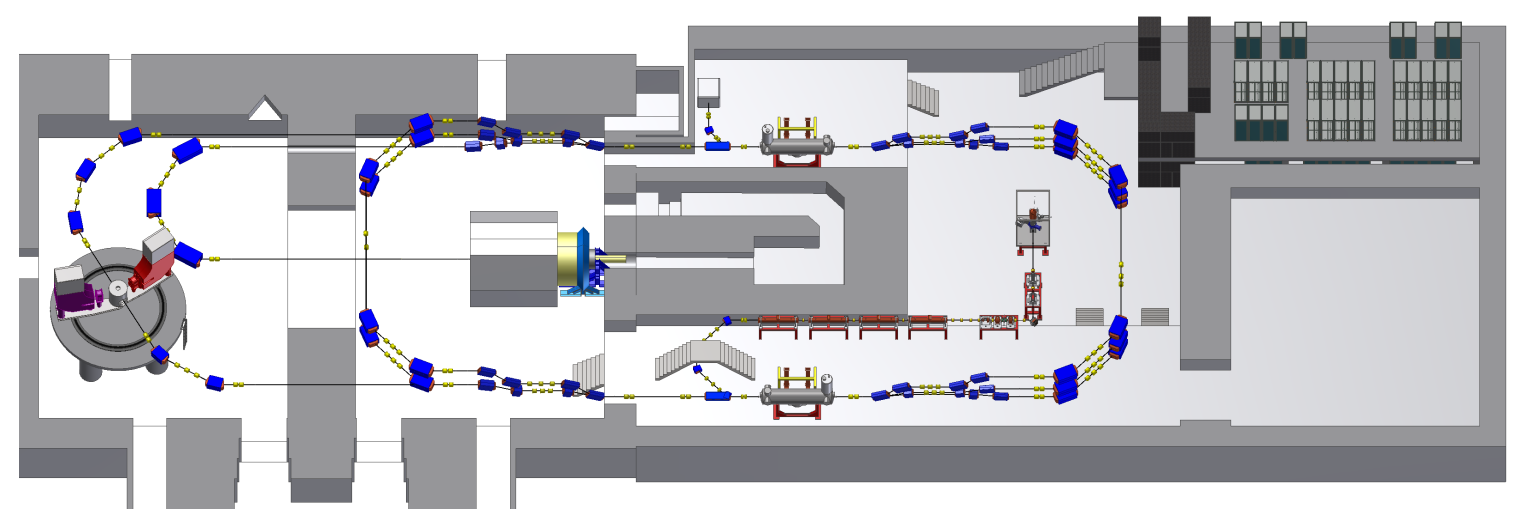

Figure 1: Layout of the new Accelerator MESA. The P2 experiment is shown in the middle, while the internal target experiment MAGIX is shown on the left. 
This beam will be used mainly for parity violating experiments, which are the most demanding experiments in respect to beam quality and stability, since asymmetries on the level of a few parts per million are the measured observables. At MESA, the P2 experiment is under construction, with the aim to measure the weak charge of the proton. For this experiment, MESA will deliver a beam energy of $155 \mathrm{MeV}$ at $1 \mathrm{~mA}$ beam current and more than $80 \%$ spin polarization with an emphasis on active beam energy, intensity, and position stabilization.

In addition to the external beam mode, MESA will be designed to be operated in energy recovery mode. In this mode, the beam will be first accelerated as in the extracted beam mode, but instead of an extraction arc the beam will be recirculated with a path length of $n+1 / 2$ wavelengths in the last arc. By this phase shift of $\lambda / 2$, the beam enters the cavities in the decelerating phase and deposes nearly all energy stored in the beam in the cavities again. This technique allows to increase the internal beam intensity to more than $10 \mathrm{~mA}$.

The energy recovery mode will be used by the MAGIX spectrometer setup for internal target experiments.

\section{The MAGIX Setup}

The general structure of electron scattering cross sections is to first order proportional to the Mott cross section, which is proportional to one over the four-momentum transfer of the exchanged virtual photon to the fourth power:

$$
\sigma \sim \frac{1}{Q^{4}}
$$

This simple formula shows the strong demands of precision experiments with electron scattering: since the four-momentum transfer is related to the electron scattering angle $\theta_{e}$ by $Q^{2}=$ $4 E E^{\prime} \sin ^{2} \frac{\theta_{e}}{2}$, a resolution for the cross section of better than $1 \%$ requires immediately an angular resolution for the electron detection of $\delta \theta<0.05^{\circ}$. Using nuclear targets, the demand of resolving energy states at a center-of-mass energy over threshold of up to $\Delta W=100 \mathrm{MeV}$ demands in addition an energy resolution of $\delta E<10^{-4}$.

These resolutions in energy and momentum can only be achieved by high resolution magnetic spectrometers. MAGIX is a setup consisting of two high resolution spectrometers with the addition of small large solid angle detectors in the target region. The spectrometers are designed as numerically optimized Quadrupole-Dipole spectrometers to achieve the required dispersion. The focal plane of the spectrometers will be equipped with tracking detectors, e.g. GEM detectors and scintillator detectors. Further particle-identification detectors are not required below pion threshold. Fig. 2 shows a CAD drawing of the current design.

In addition to the detector system, a key feature of an internal target experiment is the gas target itself. At MAGIX a super-sonic gas jet target is under construction with the possibility to be operated as cluster jet target. With this kind of target, the density can be adjust from zero to the limit given by the acceptance of the accelerator cavities in energy recovery mode.

Besides the gas jet target, an additional open tube target will be prepared for the use of polarized hydrogen as target. The density of such a target is limited by the rate of producing polarized hydrogen, e.g. in an atomic beam source or in a laser driven cell. 


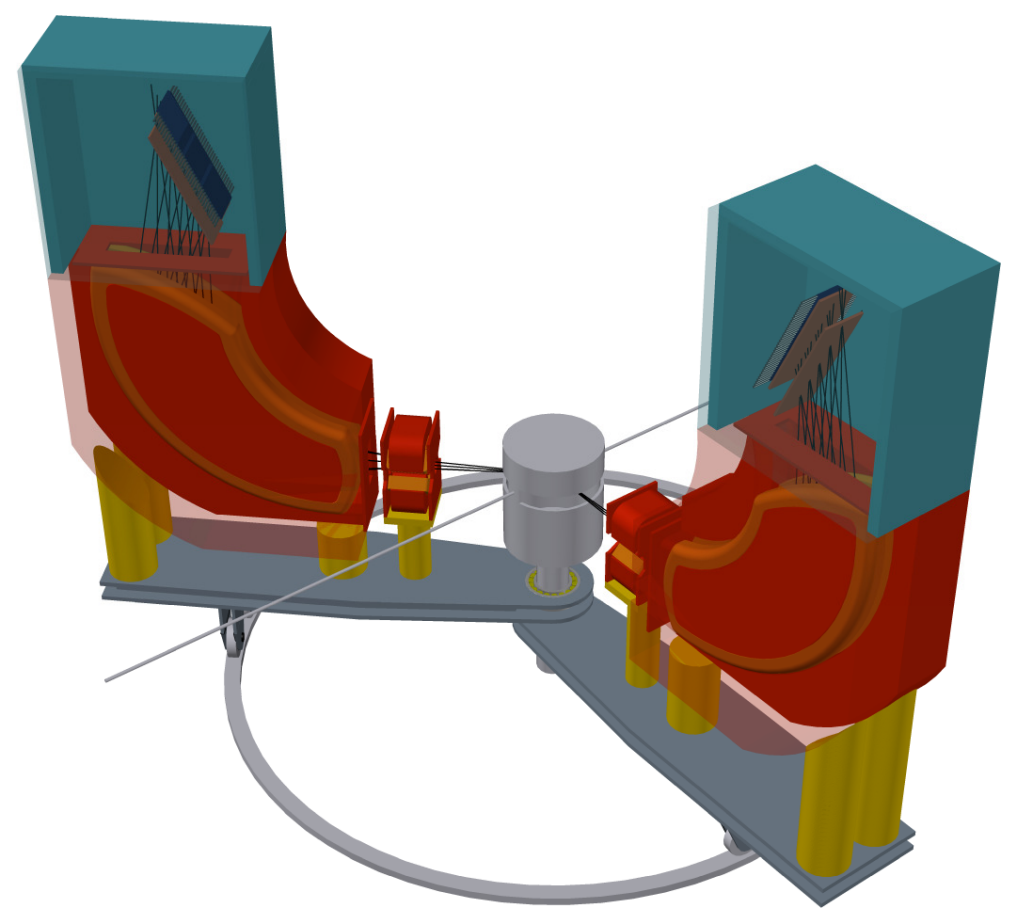

Figure 2: The MAGIX spectrometer setup.

\section{Physics Program at MAGIX}

\subsection{Magnetic radius of the Proton}

The proton radius puzzle, i.e. the disagreement between the electric radius of the proton extracted from electron scattering experiments [1] and extracted from the lamb shift of muonic atoms[2], is a well known problem of low energy physics. It is obvious, that MESA and MAGIX are well suited to measure the electric form factor of the proton at very low four-momentum transfer with high precision in the conventional way of a Rosenbluth separation. This possible experiment will not be discussed here.

In the discussion of the proton puzzle, it is often overlooked, that also the magnetic radius of the proton is a not very well known quantity. The most recent determination [1] changed the value considerably, bringing the tension in the existing data on the magnetic radius into focus.

The magnetic radius was extracted in this experiment by Rosenbluth separation of electric and magnetic form factor and the extrapolation of the magnetic form factor to zero four-momentum transfer. This technique is limited however, since the contribution of the magnetic radius to the cross section vanishes at zero. A better way to determine the magnetic form factor at low fourmomentum transfers is by double polarization experiments. These experiments are currently limited however by the minimum momentum of the recoil particle for the determination of the recoil polarization by a secondary scattering process.

Using a polarized target and a polarized has the advantage, that by the use of a gas target the full resolution can be maintained down to very low four-momentum transfer. In this case, the 
asymmetry is given by

$$
A\left(\theta^{*}, \phi^{*}\right)=A_{I} \sin \theta^{*} \cos \phi^{*}+A_{S} \cos \theta^{*}
$$

with the polarization components in dependence of the angles in respect to the virtual photon given by

$$
\begin{aligned}
& A_{I}=-2 \sqrt{\tau(1+\tau)} \tan \frac{\theta}{2} \frac{G_{E} G_{M}}{G_{E}^{2}+\left(\tau+2 \tau(1+\tau) \tan ^{2} \frac{\theta}{2}\right) G_{M}^{2}}, \\
& A_{S}=-2 \tau \sqrt{1+\tau+(1+\tau)^{2} \tan ^{2} \frac{\theta}{2}} \tan \frac{\theta}{2} \frac{G_{M}^{2}}{G_{E}^{2}+\left(\tau+2 \tau(1+\tau) \tan ^{2} \frac{\theta}{2}\right) G_{M}^{2}} .
\end{aligned}
$$

By choosing in-plane kinematics and a target polarization in direction of the virtual photon or perpendicular to this direction, the ratio

$$
\left.\begin{array}{l}
\phi^{*}=0 \\
\theta^{*}=0, \frac{\pi}{2}
\end{array}\right\} \Rightarrow A_{\perp}=\frac{A_{l}}{A_{s}} \sim \frac{G_{E}}{G_{M}}
$$

can be extracted, which is proportional to the ratio of the electric and magnetic form factors. Fig. 3 shows the result of a simulation of the MAGIX setup, assuming a polarized atomic-beam target with the density of the BLAST target as reference. Fig. 4 show the expected error bars of this simulation in comparison with existing measurements of the ration $G_{E}^{p} / G_{M}^{p}$.

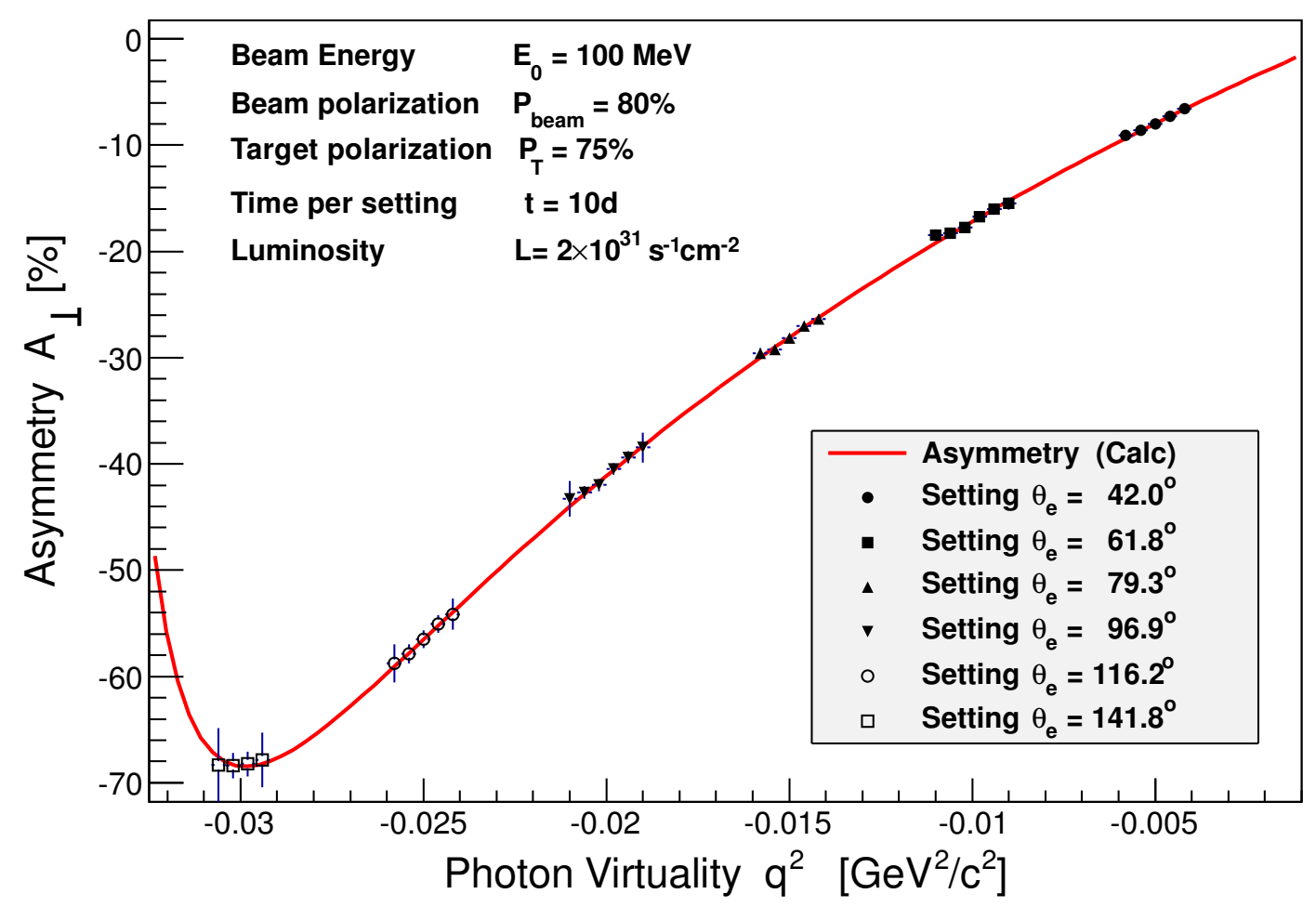

Figure 3: Simulation of a beam-target double-polarization experiment at MAGIX. Five different settings were chosen. 


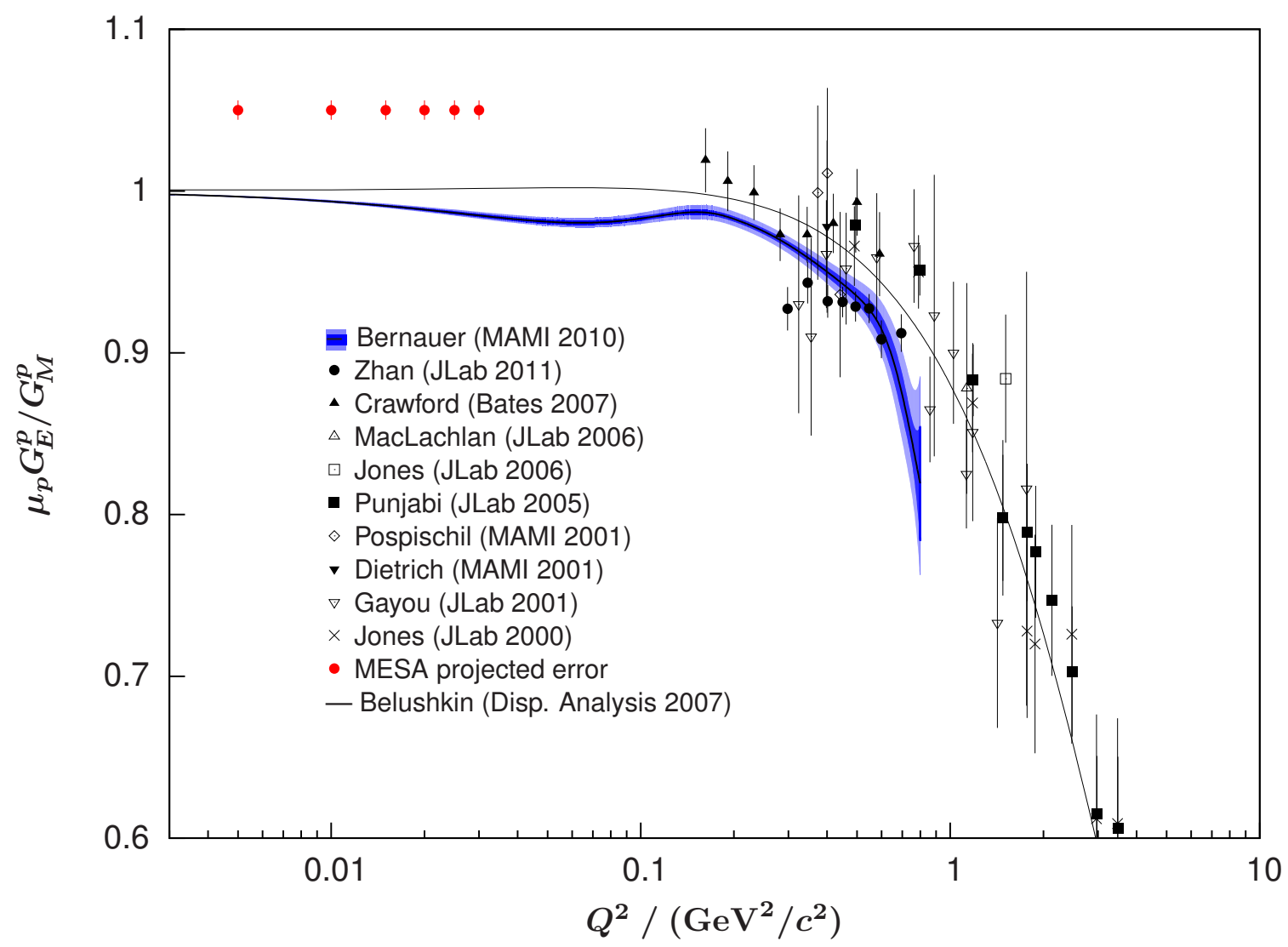

Figure 4: Expected error bars of the proposed experiment at MAGIX in comparison with existing data (see [1] for references).

\subsection{Astrophysical S-Factor}

The stellar fusion processes are the basis of all models for the abundance of elements in the universe, and by this at the core of the cosmological modeling of our universe.

These burning processes can be described mainly by nucleon and light nuclei capture cross sections. The rate of stellar burning is however extremely small since they take place at very low energies (in nuclear scales), which in general can not be reached by experiments.

Fig. 5 shows the cross section for the alpha capture on carbon ${ }^{12} C(\alpha, \gamma){ }^{16} O$, one of the key reactions of the burning chain. The region of burning in our sun (Gamow-Window) is around a center of mass energy of $E_{c . m}$. $\approx 300 \mathrm{keV}$ above threshold.

The best experiments on this reaction take place usually in underground laboratories to avoid cosmic ray background. Nevertheless a alpha particle has to be accelerated and the reaction takes place in a solid carbon target, making it necessary to extrapolate the measured cross sections to the Gamow-Window.

At MAGIX, we consider the time reversed process, ${ }^{16} O(\gamma, \alpha){ }^{12} O$, which has the same cross section (up to a phase space and spin sum factor). Instead of a real photon we will use a quasi-real photon, i.e. a photon with a very small four-momentum transfer compared to the scale set by the nuclear form factor. 
This quasi-real photon can be produced by electron-scattering, and we take advantage of the fact, that the flux of virtual photons has a pole at zero energy, enhancing the low energy region of the cross section. The second advantage of electron scattering is, that an electron is detected in coincidence with a recoil particle, i.e. the $\alpha$ particle. By this coincidence we can reduce the background contribution of cosmic rays to a negligible amount without going underground. The third advantage of this experiment is the use of a gas target, making the detection of the recoil alpha particle with silicon detectors possible. Since the energy scale is given by the detected electron, no energy resolution of the silicon detector is necessary.

First simulations show, that the count rate will be large for this process. This experiment will be governed however by the systematic errors, which are still under investigation.

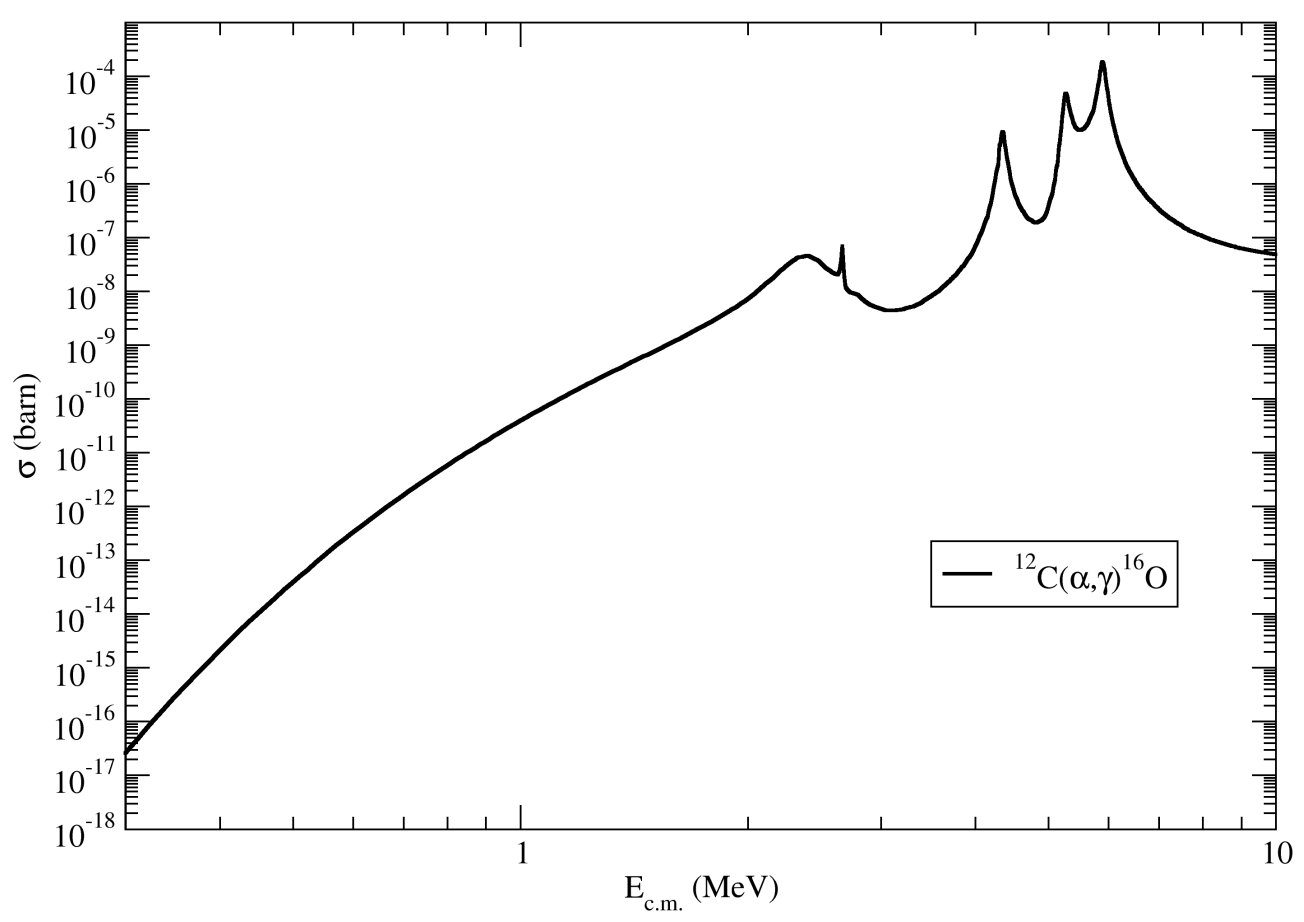

Figure 5: The cross section of ${ }^{12} C(\alpha, \gamma){ }^{16} O$. The solar burning occurs at $E_{c . m .} \approx 300 \mathrm{keV}$.

\subsection{Search for exotic particles}

Direct detection experiments for dark matter reach an incredible sensitivity nowadays, but have nevertheless not seen any clear signal up to now. In this situation, it is mandatory to extend the search for particles of the dark sector to models beyond the simple idea of a weakly interacting massive particle (WIMP). 


\subsubsection{Search for dark photons}

In Mainz we have performed already an extensive program for the search for dark photon [5]. The dark photon is one of the best motivated models, since it assumes only the existence of a "dark sector" of particles similar to the standard model[4]. In such a dark sector, also gauge bosons of the underlying interaction have to appear, in the simplest case as U(1) gauge boson, i.e. the so called dark photon.

Like MAMI, MAGIX can contribute to the exclusion limits of dark photons due to the excellent energy resolution of magnetic spectrometers, leading to an excellent mass resolution for a dark photon. In a standard experiment, the dark photon is assumed to be produced radiatively in electron scattering with a successive decay into a lepton pair, carrying the mass of the dark photon. Fig. 6 shows the exclusion limits in the low mass region which can be obtained with MAGIX.

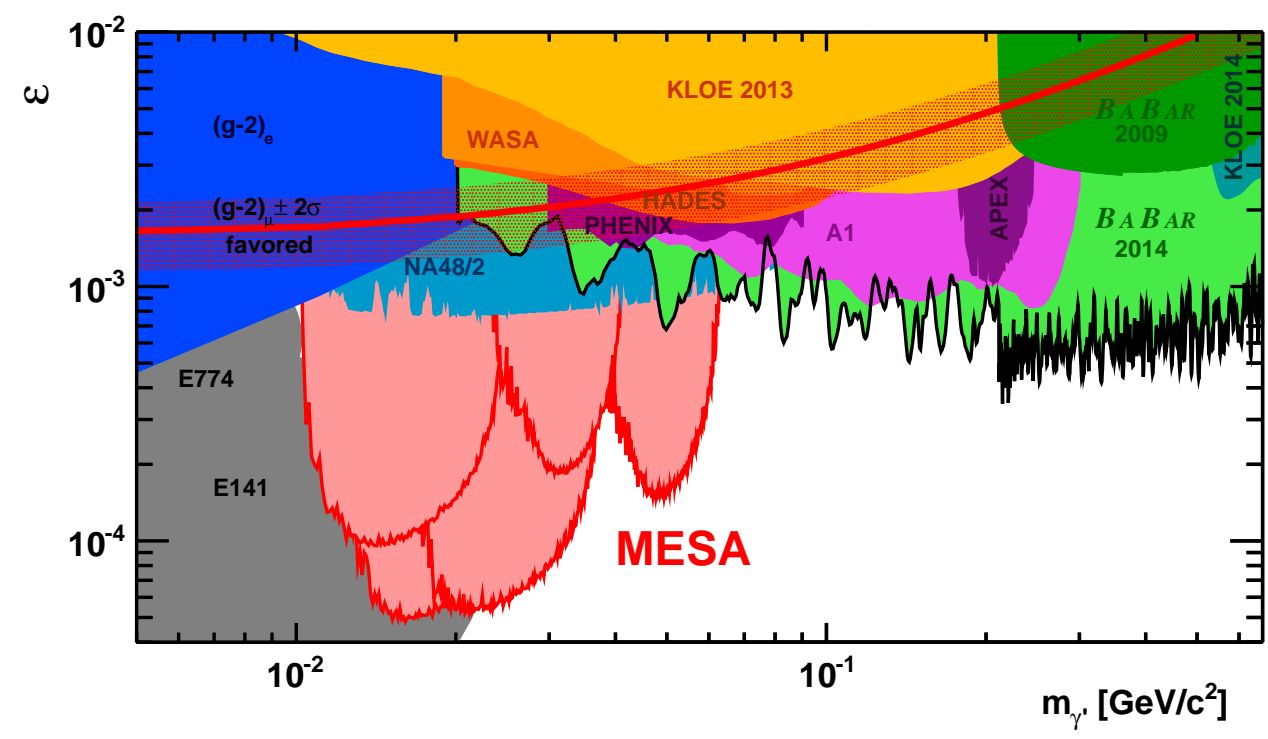

Figure 6: Exclusion limits for dark photons at MESA, compared with existing limits.

\subsubsection{Invisible decay}

Up to now, all searches for dark photon could only provide exclusion limits. One reason for this could be, that the assumption of a successive decay to a lepton pair is wrong, if the dark photon can decay e.g. into a very light particle of the dark sector. In this case the decay of the dark photon is invisible for our detectors.

MAGIX provides an unique opportunity to search also for these invisible decays. Due to the low density gas target, one can detect in addition to the scattered electron the target recoil particle and identify a possible dark photon by the missing mass. Again, the high resolution of magnetic spectrometers makes the MAGIX setup a very well suited apparatus for such a search.

\subsubsection{Beam-dump experiments}

The mentioned direct detection experiments for dark matter suffer from the need of a significant recoil of the target nucleus to produce a measurable signal. This leads to a vanishing sensitivity of these experiments in the low mass region, where no exclusion limits can be provided. 
This region, however, could be investigated by beam-dump experiments. Assuming an interaction of ordinary matter with dark matter (whatever it is), a stopped beam in a beam dump will produce dark matter particles up to half the mass given by the beam energy. A direct detection experiment positioned behind the beam dump should see now a flux of dark matter particles which now have considerable more momentum from the beam than assumed for the WIMP particles of the dark matter halo.

The sensitivity of such an experiment is now proportional to the number of particles stopped in the beam dump. MESA will run with very high current for a significant time for the parity violating experiment P2, leading to the very high yield of more than $10^{22}$ electrons on target within a few years.

The MESA halls are designed to provide the necessary space for a beam dump experiment. Fig. 7 shows a calculation by ref. [6] for the possible exclusion limits in mixing parameter $\varepsilon$ vs. mass of an interaction particle.

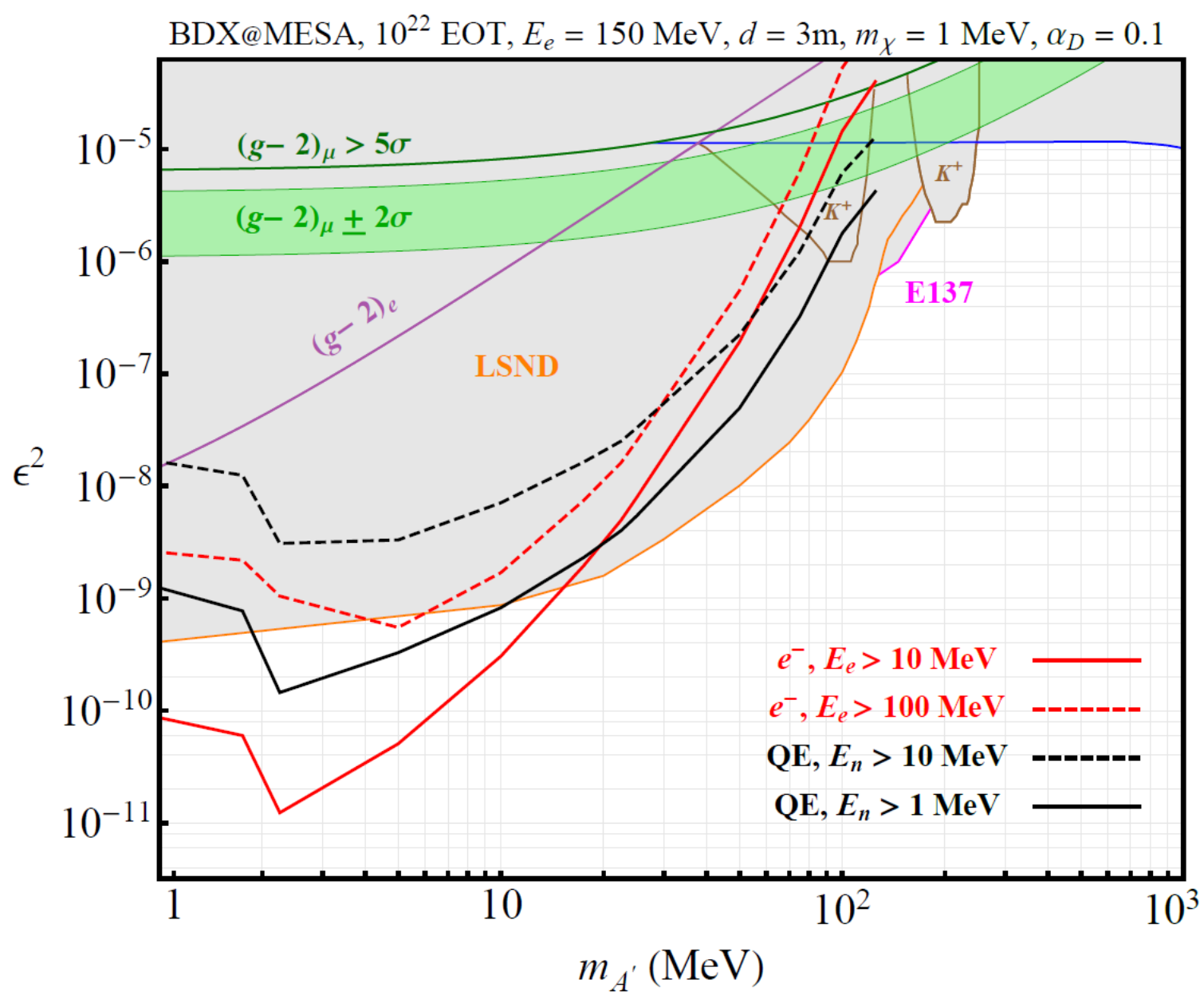

Figure 7: Exclusion limits for dark matter in a dark photon model[6] in terms of mixing parameter squared $\varepsilon^{2}$ and the mass of the dark photon. 


\section{Summary}

In this presentation, only a small part of the possible physics program at MESA could be presented. The combination of a high resolution spectrometer setup (MAGIX) with the beam intensity and quality of a state of the art energy recovery linac will open new horizons for precision measurement in the low energy region.

\section{References}

[1] Jan C. Bernauer et al., Phys. Rev. Lett. 105, 242001 (2010)

[2] R. Pohl et al., Nature 466 (2010) 213.

[3] C. Ugalde et al., JLAB Proposal PR 12-13-005

[4] N. Arkani-Hamed, et al., Phys. Rev. D 79 (2009) 015014

[5] H. Merkel et al., Phys. Rev. Lett. 112 (2014)

[6] G. Krnjaic, Perimeter Institute, private communication 